\title{
Estimating the Effect of the Red Card in Soccer When to Commit an Offense
in Exchange for Preventing a Goal Opportunity
}

\author{
Jan Vecer, Frantisek Kopriva, Tomoyuki Ichiba, \\ Columbia University, Department of Statistics, New York, NY 10027, USA
}

October 10, 2008

\begin{abstract}
We study the effect of the red card in a soccer game. A red card is given by a referee to signify that a player has been sent off following a serious misconduct. The player who has been sent off must leave the game immediately and cannot be replaced during the game. His team must continue the game with one player fewer. We estimate the effect of the red card from betting data on the FIFA World Cup 2006 and Euro 2008, showing that the scoring intensity of the penalized team drops significantly, while the scoring intensity of the opposing team increases slightly. We show that a red card typically leads to a smaller number of goals scored during the game when a stronger team is penalized, but it can lead to an increased number of goals when a weaker team is punished. We also show when it is better to commit a red card offense in exchange for the prevention of a goal opportunity.
\end{abstract}

\section{Introduction}

In this paper we study the effect of the red card in a soccer game. This problem was previously addressed by Ridder et al. (1994) using data from 3 seasons of the Dutch professional league. Their study concluded that the increase in the rate of scoring for the team that is not punished is statistically significant, but they did not observe a statistically significant decrease in the rate of scoring of the penalized team. Their approach used statistical methods to estimate the change of the rates of scoring following a red card.

A more recent and extensive study of the effect of the red card was done by Bar-Eli et al. (2006) using data from 41 seasons of the German Bundesliga. They studied the red card in relation to psychological effects. They showed that the red card reduces the scoring chances of the sanctioned team, while increasing the scoring chances for the opposite team. However, they did not look directly at the changes in the intensity of scoring which is the focus of our paper.

Previous research estimated the corresponding parameters such as probability of winning or losing the game, or the expected time to score the next goal using the entire statistical sample; thus neglecting the effects of individual situations in the games. Our approach uses a novel idea to look at the impact of a red card in each individual game, where we estimate the rates of scoring with data obtained from in-play betting markets. This enables us to incorporate more rare situations such as several red cards in a particular game. In this case the estimate from a statistical sample becomes unreliable as the number of such games is small.

It has been argued that betting markets tend to be more efficient in estimating the parameters of the models when compared to other methods. For instance, betting markets give better results when compared to opinion polls as shown by Forsythe et al. (1992).

We show in our paper that the red card leads to a fairly substantial decline in scoring intensity of the penalized team, which is in contrast with the previous study of Ridder et al. (1994), but is in accordance with Bar-Eri et al. (2006). We also show that the red card leads to a slight increase in the scoring intensity 
of the opposite team. We use data on the top international teams that played in the last World Cup and in the last Euro Cup, while the previous studies used only national league data where the dynamics of the soccer game may differ slightly.

The combined effect of the total scoring intensity depends on the relative strength of the two teams. If a stronger team is penalized, the total scoring intensity typically drops down. If a weaker team is penalized, the total scoring intensity can increase or remain the same. The decline of the total rate of scoring is typically larger when the stronger team is penalized when compared with the increase in the total scoring rate when the weaker team is penalized. However a weaker team commits a red card offense more frequently, and so the overall effect of the red card is neutral on the expected number of goals.

The last section of our paper determines when it is optimal to commit a red card offense in exchange for prevention of a possible goal opportunity for the opposite team. We assume that the objective is not to lose the game in the regulation time. This optimal time comes surprisingly early in the game. When the score is tied, and the red card offense is not followed by a penalty kick, it is better to make the offense anytime during the game when the opposing team has $57.5 \%$ or higher chances of scoring. If there is a penalty kick on the top of the red card, the optimal time to stop a certain goal is anytime after the 51st minute. When a team is leading by one goal, it is slightly less inclined to commit a red card offense. With no penalty kick, the chances of scoring must be at least $73 \%$ in order to commit such an offense. With a penalty kick, a sure goal should be stopped anytime after the 55st minute. When the team is trailing by a goal, it should become more aggressive in preventing a goal opportunity when there is no penalty kick, stopping more than $53.2 \%$ chances of scoring at any time during the game. However, the trailing team should become more conservative when a penalty kick is involved. It is never optimal to commit such an offense.

\section{Estimation of the Scoring Intensity}

We use data obtained from betting markets on the FIFA World Cup 2006 and Euro 2008. Betting markets on these two tournaments reached unprecedented efficiency and liquidity. It was also possible to buy and sell futures type contracts on the outcome of the game or the number of goals scored, and trade them even during the actual game. Prices of all traded events (win, draw or loss of a given team, and number of goals) were immediately affected by a goal or a red card.

We analyze data from the betfair.com market to estimate the implied scoring rate of a given team as the game progressed. The betting market on a given match traded the following most liquid contracts:

- Team 1 to win,

- Draw,

- Team 2 to win,

- Team 1 to score next,

- Team 2 to score next,

- No goal to be scored for the rest of the match,

- Three or more goals scored.

These contracts expired at the end of the regular time of a particular match (90 minutes + injury time). They were traded before and during the actual game. The contracts are quoted in terms of odds $1: x$. One can interpret such odds as the probability of this event. It is possible to buy the event (bet that this event will happen, or "back" it), or sell the event (bet that this event will not happen, or "lay" it). The backer of 
the event bets $\$ 1$, and receives $\$ x^{b a c k}$ (creating a total profit of $\$\left(x^{b a c k}-1\right)$ ) if the event happens, otherwise he loses his $\$ 1$ stake. The layer of the event bets $\$\left(x^{l a y}-1\right)$ and receives $\$ x^{l a y}$ (creating a total profit of $\$ 1$ ) if the event does not happen, otherwise he loses his $\$\left(x^{l a y}-1\right)$ stake. The market is set up in such a way that $x^{b a c k}<x^{l a y}$ at all times. If the event happens, the backer wins $x^{b a c k}-1$, but the layer loses a larger stake $x^{l a y}-1$. The odds $x^{\text {back }}$ and $x^{\text {lay }}$ are immediately available for backing or laying the event. One can request better odds, in which case one's order is queued in the market, and has to wait until it is matched with a counter party. However, a matching bet may never come.

\begin{tabular}{|l||r|r||r|r|}
\hline \multicolumn{1}{|c||}{} & \multicolumn{2}{c||}{ Before RC } & \multicolumn{2}{c|}{ After RC } \\
\cline { 2 - 5 } Contract & Back & Lay & Back & Lay \\
\hline Italy & 1.87 & 1.88 & 2.68 & 2.70 \\
Australia & 8.40 & 8.60 & 4.40 & 4.90 \\
The Draw & 2.78 & 2.80 & 2.42 & 2.44 \\
Italy Next Goal & 1.82 & 1.83 & 2.50 & 3.00 \\
Australia Next Goal & 6.40 & 7.00 & 3.55 & 4.20 \\
No Goal & 3.20 & 3.30 & 2.84 & 2.98 \\
Three or More Goals & 6.80 & 7.20 & 6.00 & 11.00 \\
\hline
\end{tabular}

Table 1: Odds for different betting contacts for Italy - Australia game immediately before and after the red card (Italy) in the 50th minute.

Table 1 illustrates this concept with odds taken directly before and after the moment of the red card in the Italy - Australia game. Italy was sanctioned in the 50th minute of the game. The betting market suspends all trading when there is an apparent goal or a penalty, so it is rather simple to identify the quotes that immediately precede and follow such an event. If someone were to bet $\$ 1$ on an Italian victory before the red card he would receive $\$ 1.87$ if Italy won, making a net profit of $\$ 0.87$. After the red card, the same event of an Italian victory became less likely with odds of 1:2.68. Note that the layer of the event (before the red card) had odds of $1: 1.88$, betting $\$ 0.88$ instead of $\$ 0.87$ - a gain that collects the backer of the event. This is due to the aforementioned market spread between buying and selling of an event. Note that the market spread may be relatively large if the market exhibits a small liquidity as in the case of three or more goals bet after the red card (1:6 for back and 1:11 for lay).

The market odds can serve as estimates of the likelihood of a given event. For example, the chances of Italy winning were between $1: 1.88$ and $1: 1.87$ before the penalty, which corresponds to the interval $(0.532$, $0.535)$ for the probability of this event. After the penalty, this probability dropped to the interval $(0.370$, $0.373)$.

In order to analyze the change in probabilities as they evolved during the game, especially before and after the red card event, we focus our attention on the scoring intensities of the two teams. Scoring intensities of the two teams already determine probabilities of winning, drawing or losing the game, the probability that a particular team scores next, as well as the probability of a certain number of goals. From this perspective, scoring intensities are the fundamental parameters of our analysis.

In order to find the relationship between the scoring intensities and probabilities associated with the game, we use the Poisson model for the scoring. We further assume that the scores of the two teams are independent. This is a reasonable assumption, a typical correlation of the scores of the two teams is very small. For example, the correlation of the scores in Euro 2008 tournament was only - 0.13. This model was also assumed in previous research, such as in Wesson (2002). All bets in this case depend only on two parameters: the scoring intensity of the first team $\lambda_{t}$ and the scoring intensity of the second team $\mu_{t}$ at the time $t$. Thus number of goals scored by each team follows the Poisson distribution. The market implies that 
$\lambda_{t}$ and $\mu_{t}$ are the expected number of goals to be scored by Team 1 and Team 2 respectively in the rest of the game (time between the current time $t$ and the end of the match time $T$ ).

The theoretical probabilities that correspond to the betting contracts are given by the following formulas. We assume the current score is $X_{t}: Y_{t}$ :

$$
\begin{aligned}
& \text { Win Team } 1=\mathbb{P}\left(X_{T}>Y_{T}\right)=\sum_{k=0}^{\infty} \mathbb{P}\left(X_{T}=k-X_{t}, Y_{T}<k-X_{t}\right)=\sum_{k=0}^{\infty}\left[e^{-\lambda_{t}} \frac{\lambda_{t}^{k}}{k !} \cdot \sum_{i=0}^{k+X_{t}-Y_{t}-1} e^{-\mu_{t}} \frac{\mu_{t}^{i}}{i !}\right] \\
& \text { Draw }=\mathbb{P}\left(X_{T}=Y_{T}\right)=\sum_{k=0}^{\infty}\left[e^{-\left(\lambda_{t}+\mu_{t}\right)} \cdot \frac{\lambda_{t}^{\left(k+\max \left(X_{t}+Y_{t}\right)-X_{t}\right)}}{\left(k+\max \left(X_{t}+Y_{t}\right)-X_{t}\right) !} \cdot \frac{\mu_{t}^{\left(k+\max \left(X_{t}+Y_{t}\right)-Y_{t}\right)}}{\left(k+\max \left(X_{t}+Y_{t}\right)-Y_{t}\right) !}\right] \\
& \text { Win Team } 2=\mathbb{P}\left(Y_{T}>X_{T}\right)=\sum_{k=0}^{\infty} \mathbb{P}\left(Y_{T}=k-Y_{t}, X_{T}<k-Y_{t}\right)=\sum_{k=0}^{\infty}\left[e^{-\mu_{t}} \frac{\mu_{t}^{k}}{k !} \cdot \sum_{i=0}^{k-X_{t}+Y_{t}-1} e^{-\lambda_{t}} \frac{\lambda_{t}^{i}}{i !}\right] \\
& \text { Team } 1 \text { Next Goal }=\frac{\lambda_{t}}{\lambda_{t}+\mu_{t}} \cdot\left[1-e^{-\left(\lambda_{t}+\mu_{t}\right)}\right] \\
& \text { Team } 2 \text { Next Goal }=\frac{\mu_{t}}{\lambda_{t}+\mu_{t}} \cdot\left[1-e^{-\left(\lambda_{t}+\mu_{t}\right)}\right] \\
& \text { No Goal }=e^{-\left(\lambda_{t}+\mu_{t}\right)} \\
& \text { Three+ }=\mathbb{P}\left(X_{T}+Y_{T} \geq 3\right)=\sum_{k=\left(3-X_{t}-Y_{t}\right)^{+}}^{\infty} e^{-\left(\lambda_{t}+\mu_{t}\right)} \frac{\left(\lambda_{t}+\mu_{t}\right)^{k}}{k !}
\end{aligned}
$$

Note that we have 7 contracts that depend only on two parameters $\lambda_{t}$ and $\mu_{t}$, and so this problem is over-parameterized. The market may even admit arbitrage (risk free profit) if the contract prices are not properly related to each other. For instance, the probabilities of Win of Team 1, Draw, and Win of Team 2 should add up to 1 . However, due to the inefficiencies of the market, such as the bid-ask spread, asynchronous trading, and market order delay, it may be difficult to lock into these opportunities.

Let $x^{\text {back }}$ be the odds of backing a specific event, and let $p$ be the theoretical probability of this event. Then the return of this bet is given by

$$
x^{b a c k} \cdot p-1
$$

For instance if one was to bet on an outcome of a fair coin toss $\left(p=\frac{1}{2}\right)$, the return would be zero if $x^{b a c k}=2$. If the odds were higher than 2 , than the return would be positive, if the odds were smaller, than the return would be negative. For example when $x^{\text {back }}=2.02$, the return is $1 \%$, so one should expect to earn on average $\$ 1$ per $\$ 100$ invested. If the odds were $x^{b a c k}=1.98$, the return is $-1 \%$, so one should expect to lose on average $\$ 1$ per $\$ 100$ invested.

The return for the laying of an event is calculated in a slightly different way because it requires a bet of $\$\left(x^{l a y}-1\right)$ in order to win $\$ 1$. The return is given by

$$
\frac{x^{\text {lay }}}{x^{\text {lay }}-1} \cdot(1-p)-1
$$

Given a fair coin toss, the return is zero when $x^{l a y}=2$. The return is positive when the odds are smaller, and is negative when the odds are larger. For example, when $x^{l a y}=1.98$, the corresponding return is $1.02 \%$, when $x^{\text {lay }}=2.02$, the corresponding return is $-0.98 \%$. 
In order to estimate $\lambda_{t}$ and $\mu_{t}$ from the odds quoted by the betting market, we choose $\hat{\lambda}_{t}$ and $\hat{\mu}_{t}$ that minimizes the best expected return of all contracts combined, namely finding a minimizer of the following formula:

$$
\min _{\lambda_{t}, \mu_{t}} \max _{i}\left(x_{i}^{b a c k} \cdot \mathbb{P}_{i}\left(\lambda_{t}, \mu_{t}\right)-1 ; \frac{x_{i}^{l a y}}{x_{i}^{\text {lay }}-1} \cdot\left(1-\mathbb{P}_{i}\left(\lambda_{t}, \mu_{t}\right)\right)-1\right),
$$

where $\mathbb{P}_{i}\left(\lambda_{t}, \mu_{t}\right)$ is the probability of $i$-th event given the intensities of scoring $\lambda_{t}$ and $\mu_{t}$. The properties of the min-max estimator are discussed in Cox and Hinkley (1979).

\begin{tabular}{|l|r|r|r||r|r|}
\hline & & & \multicolumn{2}{|c||}{ Return } \\
\cline { 5 - 6 } Contract & $1 / x^{\text {lay }}$ & $1 / x^{\text {back }}$ & $\mathbb{P}_{i}\left(\hat{\lambda}_{t}, \hat{\mu}_{t}\right)$ & Back & Lay \\
\hline Italy & 0.532 & 0.535 & 0.534 & -0.002 & -0.004 \\
Australia & 0.116 & 0.119 & 0.099 & -0.171 & $\mathbf{0 . 0 2 0}$ \\
The Draw & 0.357 & 0.360 & 0.367 & $\mathbf{0 . 0 2 2}$ & -0.016 \\
Italy Next Goal & 0.546 & 0.549 & 0.560 & $\mathbf{0 . 0 2 0}$ & -0.031 \\
Australia Next Goal & 0.143 & 0.156 & 0.152 & -0.030 & -0.010 \\
No Goal & 0.303 & 0.313 & 0.288 & -0.079 & $\mathbf{0 . 0 2 2}$ \\
Three or More Goals & 0.139 & 0.147 & 0.130 & -0.113 & $\mathbf{0 . 0 1 0}$ \\
\hline
\end{tabular}

Table 2: Probabilities implied by the betting odds for the Italy - Australia game right before the time of the red card in the 50th minute (columns $1 / x^{\text {lay }}, 1 / x^{b a c k}$ ), probabilities of these events using a Poisson model with the optimal choice of parameters $\hat{\lambda}_{t}=0.980$ and $\hat{\mu}_{t}=0.265$ (column $\left.\mathbb{P}_{i}\left(\hat{\lambda}_{t}, \hat{\mu}_{t}\right)\right)$, and returns that correspond to the buying or selling these events. Positive returns are typed in bold.

Table 2 illustrates the choice of the optimal parameters for the Italy - Australia game right before the moment of the red card in the 50th minute. It not only shows the probabilities implied by the betting odds, but also the theoretical probability of any event that come with the optimal choice of the scoring parameters $\hat{\lambda}_{t}=0.980$ and $\hat{\mu}_{t}=0.265$. Note that some of the betting contracts yield a positive return for this choice of parameters (such as backing The Draw, or Italy Next Goal, or laying Australia, No Goal, and Three or More Goals), but the best expected return is $2.2 \%$, which is well below the standard commission rate of $5 \%$ charged by the market. The optimal choice of the scoring intensities keeps the best return at the lowest level, because for any other choice of intensities this discrepancy is larger. Although we find some contracts with positive return, it is still not clear whether they should be bought or sold. Their price looks favorable with respect to the prices of other betting contracts, but if taken alone, they could still be quoted at a fair price.

After the red card, the scoring rate for Italy fell from 0.980 to 0.677 , while the scoring rate for Australia rose from 0.265 to 0.477 .

It is possible to use different estimators of $\lambda_{t}$ and $\mu_{t}$. One example is the $L^{2}$ least square estimator

$$
\min _{\lambda_{t}, \mu_{t}} \sum_{i}\left[\left(\frac{1}{x_{i}^{\text {back }}}-\mathbb{P}_{i}\left(\lambda_{t}, \mu_{t}\right)\right)^{2}+\left(\frac{1}{x_{i}^{\text {lay }}}-\mathbb{P}_{i}\left(\lambda_{t}, \mu_{t}\right)\right)^{2}\right],
$$

another one is the $L^{1}$ estimator:

$$
\min _{\lambda_{t}, \mu_{t}} \sum_{i}\left[\left|\frac{1}{x_{i}^{\text {back }}}-\mathbb{P}_{i}\left(\lambda_{t}, \mu_{t}\right)\right|+\left|\frac{1}{x_{i}^{\text {lay }}}-\mathbb{P}_{i}\left(\lambda_{t}, \mu_{t}\right)\right|\right]
$$

For comparison, $L^{2}$ estimators for the Italy - Australia game prior to the red card are $\hat{\lambda}_{t}^{2}=0.959$ and $\hat{\mu}_{t}^{2}=0.265, L^{1}$ estimators are $\hat{\lambda}_{t}^{1}=0.948$ and $\hat{\mu}_{t}^{1}=0.263$, which are close enough to the minmax estimator 
$\hat{\lambda}_{t}=0.980$ and $\hat{\mu}_{t}=0.265$. The advantage of the minmax estimator is that it is more robust when there is a significant liquidity gap, that is, when some of the discrepancies between $\frac{1}{x_{i}}$ and $\mathbb{P}_{i}\left(\lambda_{t}, \mu_{t}\right)$ may become so large that they can affect an estimate that is based on $L^{1}$ or $L^{2}$. Minmax estimators are not susceptible to such effects.

Figures 1-3 show the market implied intensities using the minmax estimator for selected games from the World Cup to illustrate this concept. We start our inference analysis at the beginning of the game (time $t=0$ ), and finish when the game ends in the regulation (90 minutes) plus injury time. We indicate the break between the two halves, which lasts 15 minutes.

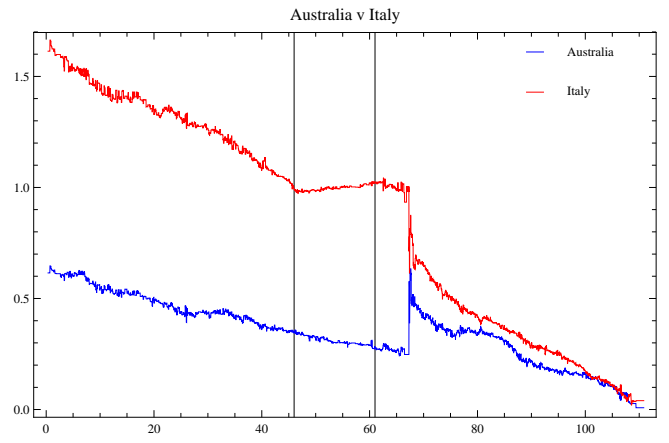

Figure 1: Implied scoring intensities in the Italy - Australia game. A red card was given to Italy in the 50th minute of the game.

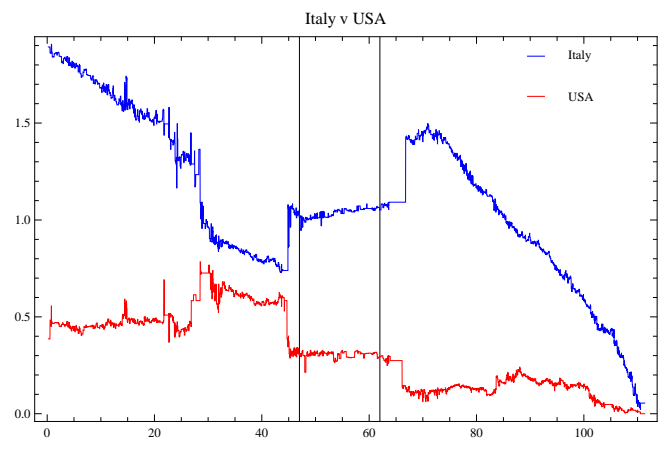

Figure 2: Implied scoring intensities in the Italy - USA game. Italy was sanctioned with a red card in the middle of the first half $\left(25^{\prime}\right)$, followed by a red card given to the US team at the end of the first half (45'), and the beginning of the second half $\left(48^{\prime}\right)$.

\section{Estimating the Effect of the Red Card}

Upon receiving a red card the sanctioned team must complete the rest of the match with one player less. During the FIFA World Cup 2006 tournament, 28 players in total were sanctioned. During Euro 2008 tournament 3 players received a red card. Our analysis includes a total of 27 cases of red cards; 2 happened in overtime, and 2 at the very end of the game. We use the method described in the previous section and estimate the scoring intensities of both teams before and after a red card. Multiple red cards were given in 


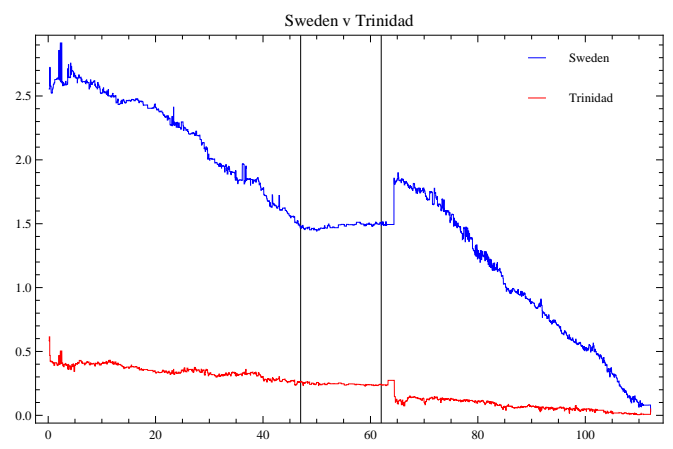

Figure 3: Implied scoring intensities in the Trinidad - Sweden game. Trinidad was sanctioned by a red card in the early stages of the second half of the game $\left(46^{\prime}\right)$.

4 games; 2 red cards in the Cote d'Ivoire - Serbia game, 3 red cards in Italy - USA and Croatia - Australia games and 4 red cards in the Portugal - Holland game.

The scoring rate of the sanctioned team is denoted by $\lambda$, with $\lambda^{\text {old }}$ being the rate prior to the red card, and $\lambda^{\text {new }}$ being the rate after the red card. Similarly, the rates of the opposing team are denoted by $\mu$, with $\mu^{o l d}$ being the rate prior to the red card and $\mu^{\text {new }}$ being the rate after the red card. The complete list of these rates of scoring is given in Table 3 .

In order to predict the change in rates after the red card event we use the following regression model:

$$
\lambda^{\text {new }}=\theta_{1} \cdot \lambda^{\text {old }}+\epsilon_{1}
$$

for a certain $\theta_{1}$ for the team which was just penalized, and

$$
\mu^{\text {new }}=\theta_{2} \cdot \mu^{\text {old }}+\epsilon_{2}
$$

for a certain $\theta_{2}$ for the opposite team. The error terms in the model reflect individual situations within each game such as the suspension of a key player.

Linear regression leads to the following estimates:

$$
\hat{\theta}_{1}=0.663 \approx \frac{2}{3}
$$

with $R^{2}=0.972$, and

$$
\hat{\theta}_{2}=1.237 \approx \frac{5}{4}
$$

with $R^{2}=0.991$. Thus the scoring intensity of the penalized team drops approximately to $\frac{2}{3}$ of the intensity that precedes the red card, and the scoring intensity of the opposing team increases approximately to $\frac{5}{4}$ of the original intensity before the red card. The graph that illustrates the change of intensity of the penalized team is given in Figure 4 and the graph that illustrates the change of intensity of the opposing team is given in Figure 5.

\section{Red Card and Expected Number of Goals}

This section discusses the question whether we are expected to see more or less goals after a red card. The total scoring intensity of both teams combined before the red card is given by

$$
\lambda^{\text {old }}+\mu^{\text {old }}
$$




\begin{tabular}{|c|c|c|c|c|c|c|}
\hline Game & Red Card & Time & $\lambda^{\text {old }}$ & $\lambda^{\text {new }}$ & $\mu^{\text {old }}$ & $\mu^{\text {new }}$ \\
\hline Trinidad - Sweden & Trinidad & $46^{\prime}$ & 0.224 & 0.048 & 1.501 & 1.741 \\
\hline Korea - Togo & Togo & 53 ' & 0.350 & 0.313 & 0.702 & 1.030 \\
\hline Spain - Ukraine & Ukraine & 47 ' & 0.430 & 0.244 & 0.728 & 0.858 \\
\hline Germany - Poland & Poland & $75^{\prime}$ & 0.144 & 0.083 & 0.608 & 0.751 \\
\hline Argentina - Serbia & Serbia & $65^{\prime}$ & 0.300 & 0.227 & 0.865 & 0.941 \\
\hline Mexico - Angola & Angola & $79^{\prime}$ & 0.111 & 0.050 & 0.480 & 0.519 \\
\hline Italy - USA & Italy & $28^{\prime}$ & 1.272 & 0.846 & 0.381 & 0.567 \\
\hline Italy - USA & USA & $45^{\prime}$ & 0.563 & 0.325 & 0.769 & 1.037 \\
\hline Italy - USA & USA & $47^{\prime}$ & 0.296 & 0.099 & 1.092 & 1.415 \\
\hline Czech & eech & $65^{\prime}$ & 0.740 & 0.538 & 0.364 & 0.477 \\
\hline Cote d'? & Serbia & $46^{\prime}$ & 0.615 & 0.561 & 1.093 & 1.478 \\
\hline Cote & e d'Ivoire & $92 '$ & -- & --- & -- & --- \\
\hline Mexico - P & exico & $61^{\prime}$ & 0.567 & 0.399 & 0.559 & 0.628 \\
\hline Italy - Czech & Czech & $47^{\prime}$ & 0.715 & 0.468 & 0.968 & 1.303 \\
\hline Croatia - A & Croatia & $85^{\prime}$ & 0.174 & 0.106 & 0.194 & 0.271 \\
\hline & & 87 ' & 0.256 & 0.189 & 0.069 & 0.140 \\
\hline Cro & & 93 & 0.088 & 0.110 & 0.115 & 0.065 \\
\hline $\mathrm{U}$ & & 46 & 0.406 & 0.202 & 1.064 & 1.285 \\
\hline Ger & & $35^{\prime}$ & 0.547 & 0.493 & 1.054 & 1.306 \\
\hline & & $46^{\prime}$ & 0.479 & 0.286 & 0.825 & 0.983 \\
\hline & & $63{ }^{\prime}$ & 0.997 & 0.618 & 0.251 & 0.372 \\
\hline & & & 0.245 & 0.153 & 0.427 & 0.523 \\
\hline & land & $95^{\prime}$ & 0.048 & 0.046 & 0.038 & 0.036 \\
\hline & & 50 ' & 0.980 & 0.677 & 0.265 & 0.477 \\
\hline Brazil - & & $81 '$ & 0.141 & 0.020 & 0.401 & 0.628 \\
\hline England - Portu & gland & $62 '$ & 0.592 & 0.272 & 0.321 & 0.436 \\
\hline Croatia - Germany & & $92^{\prime}$ & & & & -- \\
\hline Turkey - Czech & & $92^{\prime}$ & 0.079 & 0.051 & 0.064 & 0.101 \\
\hline France - Italy & France & $24{ }^{\prime}$ & 1.012 & 0.654 & 1.194 & 1.300 \\
\hline
\end{tabular}

Table 3: Rates of scoring of the sanctioned and the opposing team immediately before and immediately after the red card. The table contains 26 games from the World Cup 2006 (top), and 3 games from the Euro 2008 (bottom). In 2 cases the red card happened at the very end of the game.

and after the red card this expression becomes

$$
\lambda^{\text {new }}+\mu^{\text {new }} .
$$

We want to find condition when

$$
\lambda^{\text {new }}+\mu^{\text {new }} \geq \lambda^{\text {old }}+\mu^{\text {old }} .
$$

Since $\lambda^{\text {new }} \approx \frac{2}{3} \lambda^{\text {old }}$, and $\mu^{\text {new }} \approx \frac{5}{4} \mu^{\text {old }}$, the above inequality is approximately valid when

$$
\mu^{\text {old }} \geq \frac{4}{3} \lambda^{\text {old }} .
$$

Thus one should expect more goals if a weaker team is penalized, but fewer goals if a stronger or comparable team is penalized. Figure 6 conveys this situation. It confirms that when a stronger or comparable team is penalized, the decline of the total scoring rates is visible in most of such games. The stronger team that is penalized loses more offensive power in comparison with its defensive capacities, and although the weaker team improves its scoring rate, this increase is smaller. However, when a weaker team is penalized, the total scoring rate sometimes increases, but sometimes it stays the same. 


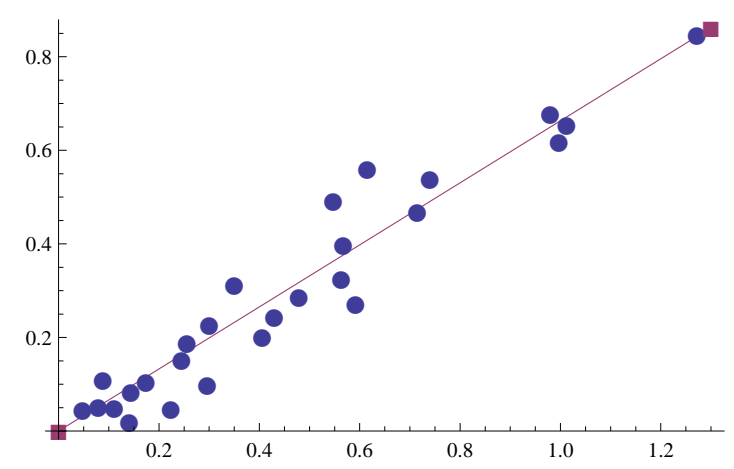

Figure 4: Relationship between the scoring intensity prior to the red card (x-axis) and after the red card (y-axis) for the sanctioned team. The line represents the best linear fit with slope 0.663 .

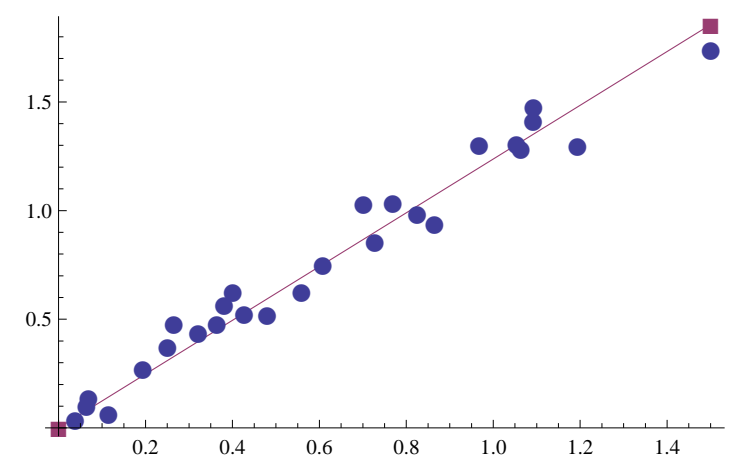

Figure 5: Relationship between the scoring intensity prior to the red card ( $\mathrm{x}$-axis) and after the red card (y-axis) for the opposite team. The line represents the best linear fit with slope 1.237.

\section{$5 \quad$ Red Card or Goal}

Consider the situation where a player can prevent a goal with the help of misconduct and hence with the risk of expulsion. Such situations occur quite often, such as when a goalie trips a player of the opposing team in when he is in a favorable scoring position, or when a defender stops a ball flying into an empty goal with his hands. When the game is tied and is approaching its end, it is better to commit such an offense, even in the situation where a red card and a penalty kick are certain. Otherwise his team will lose the game anyway. On the other hand, it may not be optimal to commit a red card offense in the early stages of the game, since there would be enough time to score and come back into the game.

We consider the question: At what point in the game is better to commit a red card offense in exchange for preventing a goal opportunity for the opposing team? This optimal time depends on the current score and the objective of the team. Let us assume that the objective of the team is not to lose the game during the regulation time and that the current score is either tied, or one of the teams leads by one goal. We look at two situations: a red card is issued with a penalty kick, or a red card is issued without a penalty kick. We further assume that the two teams are of comparable strength, with the average scoring rate at 1.1 goals per game.

It has been observed in the previous research, such as in Garicano and Palacios-Huerta (2005), that soccer teams often choose to prevent goal opportunities in exchange for illegal offenses. In our study we show that such behavior, while unsportsmanlike, can be optimal to achieve a victory or a tie in a given situation. One 


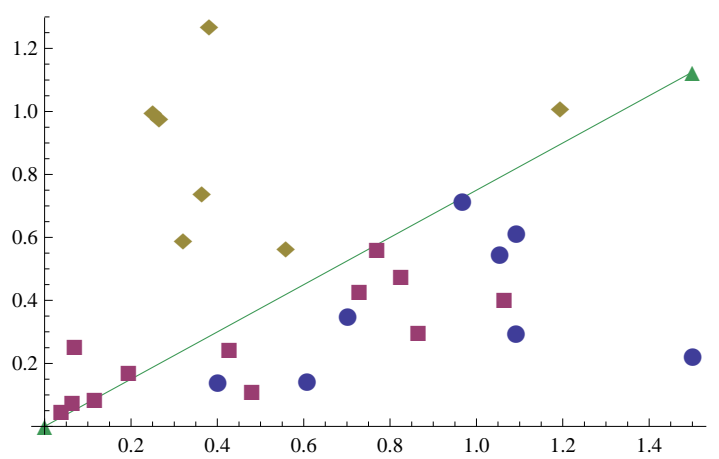

Figure 6: Change of the overall scoring rate given the relative strengths of the two teams: the $\mathrm{x}$-axis represents the scoring intensity of the opposing team prior to a red card $\left(\mu^{\text {old }}\right)$, the $\mathrm{y}$-axis represents the scoring intensity of the penalized team prior to a red card $\left(\lambda^{\text {old }}\right)$. The line $\lambda^{\text {old }}=\frac{3}{4} \mu^{\text {old }}$ should separate games with an increased scoring intensity (below the line - when a weaker team is sanctioned) from games with a reduced scoring intensity (above the line - when a stronger or a comparable team is sanctioned). Circle points represent games with an increased scoring intensity that are located below the line, but they are mixed with square points that represent games with no change of overall scoring intensity. Diamond points represent games with a reduced scoring intensity that are located above the line.

of the reasons for this is that a soccer game has a relatively low number of goals, and thus a single goal often makes a difference in the final outcome.

Taking into account the situation when the score is tied, Figure 7 shows optimal time when it is better to commit a red card offense resulting in a penalty kick as a function of the probability of scoring by the opposite team. We assume an $80 \%$ success rate of scoring from a penalty kick. Obviously, if the current chance of scoring is less than $80 \%$, it is better to let it play since a penalty kick would create a more favorable situation for the opposing team. However, if the chance of scoring is higher, it is sometimes optimal to commit the offense. Notably, when the chance of scoring is $100 \%$ (for example, when the ball is flying in an empty goal), it is better to stop it in the 51st minute of the game.

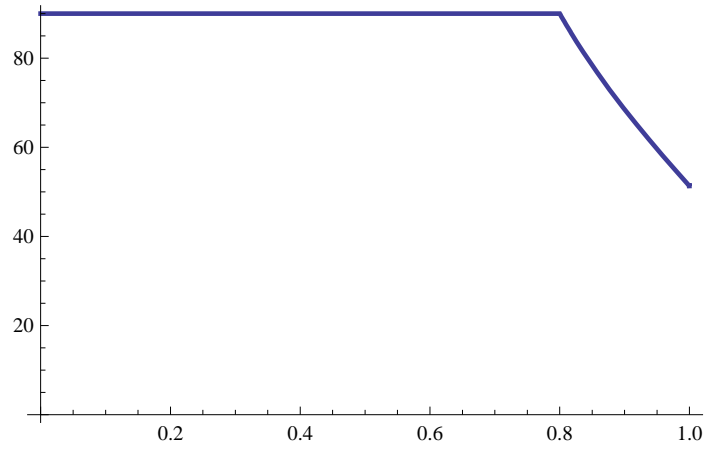

Figure 7: Optimal time for a red card offense resulting in a penalty kick as a function of the probability of scoring a goal in a given situation assuming the current score is tied.

When the violation does not lead to a penalty kick, but only a red card, optimal time to commit such an offense comes even earlier in the game. When the chance of scoring is just at $57.5 \%$ or higher, it is better to prevent such a scoring opportunity from the very beginning of the game as seen in Figure 8. 


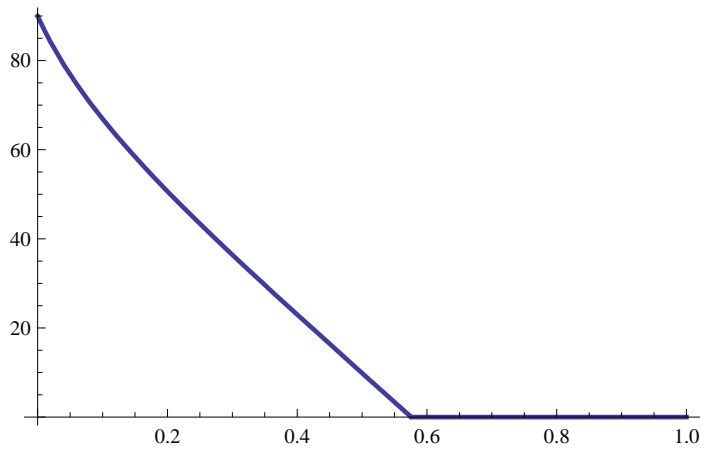

Figure 8: Optimal time for a red card offense that does not result in a penalty kick as a function of the probability of scoring a goal in a given situation assuming the current score is tied.

Figure 9 captures the optimal time to commit a red card offense resulting in a penalty kick when the sanctioned team is leading by a goal and when the objective is not to lose the game. The team should be more conservative for such a violation since the one goal lead provides a certain cushion. If the chances of scoring are below $86.6 \%$, the team should let it play out. In the situation where the goal is certain if not disrupted, the optimal time to commit an offense is anytime after the 55th minute, a little later than in the case when the game is tied.

When the offense does not lead to a penalty kick, it is optimal to let the game go if the chance of scoring is below $33.4 \%$, but to commit such an offense anytime during the game when the chance is above $73 \%$ as seen in Figure 10. Thus when the team is leading by a goal, it is less inclined to commit a red card offense when compared to the tied score situation.

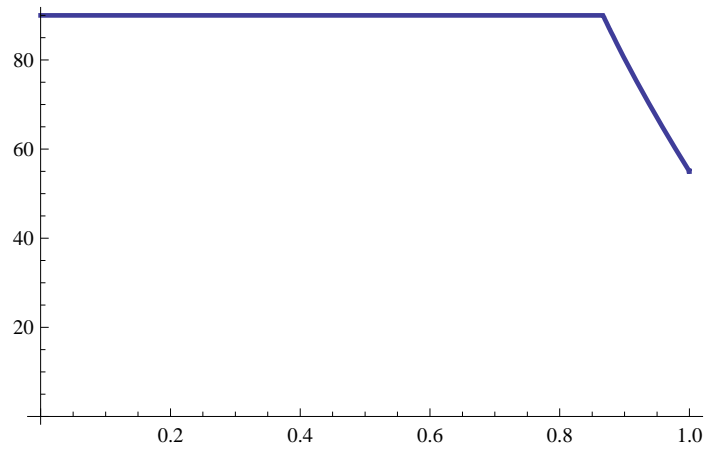

Figure 9: Optimal time for a red card offense resulting in a penalty kick as a function of the probability of scoring a goal in a given situation assuming team committing the offense is leading by one goal.

An interesting situation occurs when the team who commits a red card offense is trailing by one goal. That team should never risk an offense that results a penalty kick. It is optimal that the opposing team scores with high probability, but leaves the trailing team with the full number of players. As seen in Figure 11, the optimal time to commit such an offense is at the end of the game. However, the trailing team has to be more aggressive in stopping the scoring chance when there is no risk of a penalty kick as seen in Figure 12. When the chance of scoring is just $53.2 \%$ or higher, it is optimal to stop such action from the very beginning of the game when the penalized team is trailing by a goal. This should be compared to the committing an 


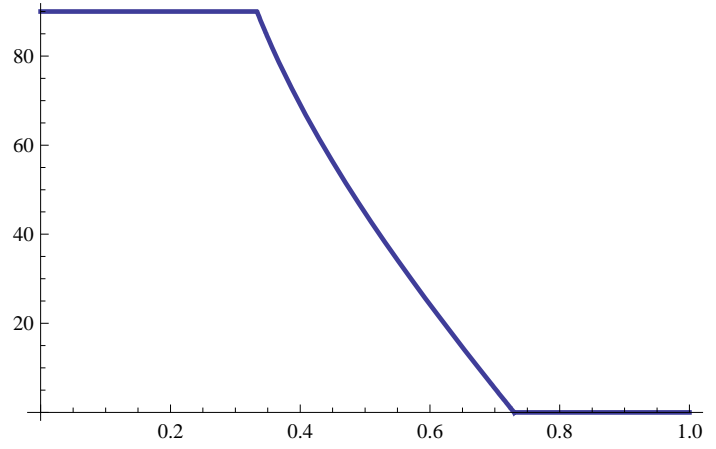

Figure 10: Optimal time for a red card offense that does not result in a penalty kick as a function of the probability of scoring a goal in a given situation assuming team committing the offense is leading by one goal.

offense anytime during the game with a $57.5 \%$ chance of scoring assuming the score is tied.

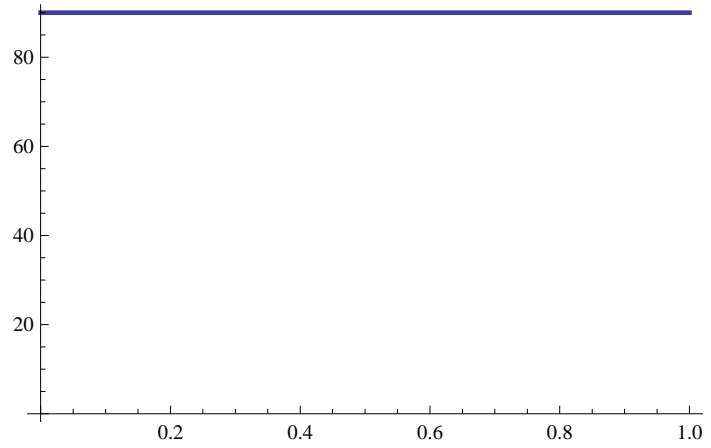

Figure 11: Optimal time for a red card offense resulting in a penalty kick as a function of the probability of scoring a goal in a given situation assuming the team committing the offense is trailing by one goal. Note that the optimal time is the end of the game in all situations.

\section{Conclusion}

We have introduced a new method for estimating the scoring intensity in a soccer game using data from in play betting markets. We have shown that when one of the teams receives a red card, its scoring intensity is reduced to about $\frac{2}{3}$ of the original intensity, whereas the intensity of the opposing team increases by a factor of about $\frac{5}{4}$. This observation has allowed us to study the effect of the combined scoring intensity, and conclude that the expected number of goals decreases when a stronger or comparable team is penalized, while the expected number of goals can increase or stay the same when a weaker team is penalized. We have also shown when it is optimal to stop a scoring opportunity at the expense of a sanction (red card or a penalty kick). The optimal time depends on the score. When the team is leading by a goal, it becomes less inclined to commit a red card offense when compared to the tied score situation. When the team is trailing by a goal, it becomes more cautious when it comes to a situation that involves a red card resulting in a penalty kick, but more aggressive when only a red card sanction is involved. 


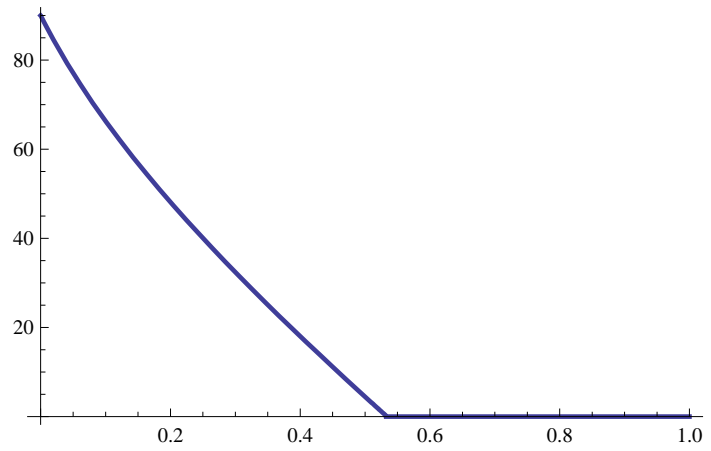

Figure 12: Optimal time for a red card offense that does not result in a penalty kick as a function of the probability of scoring a goal in a given situation assuming the team committing the offense is trailing by one goal.

\section{References}

[1] Bar-Eli, M., G. Tenenbaum, S. Geister, "Consequences of Players' Dismissal in Professional Soccer: A Crisis Related Analysis of Group Size Effect," Journal of Sport Sciences, 1083-1094, 2006.

[2] Cox, D., D. Hikley, "Theoretical Statistics," Chapman - Hall, 1979.

[3] Forsythe, R., F. Nelson, G. R. Neumann, J. Wright, "Anatomy of an Experimental Political Stock Market," The American Economic Review, Vol. 82, No. 5, 1142-1161, 1992.

[4] Garicano, L., I. Palacios-Huerta, "Sabotage in Tournaments: Making the Beautiful Game a Bit Less Beautiful." Working Paper, 2005.

[5] Ridder, G., J. S. Cramer, P. Hopstaken, "Down to Ten: Estimating the Effect of a Red Card in Soccer," JASA, Vol. 89, No. 427, 1994.

[6] Wesson, J., The Science of Soccer, IoP, 2002. 